\title{
Ocrelizumab-induced inflammatory bowel disease-like illness characterized by esophagitis and colitis
}

\author{
Alex Barnes $^{a, b}$, Dirk Hofmann ${ }^{a, b, d}$, Lesley-Ann Hall ${ }^{b, e}$, Sonja Klebe ${ }^{b, c}$, Réme Mountifield $^{a, b}$ \\ Flinders Medical Centre, South Australia, Australia
}

Abstract

Ocrelizumab is an intravenous anti-CD20 monoclonal antibody, approved for use in primary progressive multiple sclerosis due to its selective depletion of B-lymphocytes. Herein we describe the case of a 56-year-old female who developed odynophagia and bloody diarrhea following treatment with ocrelizumab. This was characterized endoscopically by ulcerations in the esophagus and colon. The patient was treated with high-dose intravenous glucocorticoids with good clinical response.

Keywords Ocrelizumab, inflammatory bowel disease, immunotherapy, colitis, esophagitis

Ann Gastroenterol 2021; 35 (1): 1-2

\section{Introduction}

Ocrelizumab is a humanized anti-CD20 monoclonal antibody shown to improve clinical and radiological outcomes in relapsing multiple sclerosis [1]. It is currently the only approved therapeutic agent shown to slow disability progression in primary progressive multiple sclerosis [2]. Ocrelizumab acts to selectively deplete B-lymphocytes, mimicking the action of the alternative anti-CD20 monoclonal antibody rituximab, with which it shares an overlapping epitope. Its potential benefit relates to decreased immunogenicity allowing for prolonged and effective treatment. Known adverse effects include infusion-related reactions and an increased incidence of serious infections. To our knowledge, a single case of ocrelizumab-induced colitis [3] has been reported, with no cases of esophagitis previously reported.

\section{Case report}

A 56-year-old female patient with a 6-year history of primary progressive multiple sclerosis presented to our facility with a

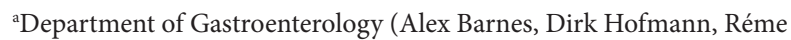
Mountifield); ${ }^{\mathrm{b} C o l l e g e ~ o f ~ M e d i c i n e ~ a n d ~ P u b l i c ~ H e a l t h ~(A l e x ~ B a r n e s, ~}$ Dirk Hofmann, Lesley-Ann Hall, Sonja Klebe, Réme Mountifield); 'Department of Anatomical Pathology (Sonja Klebe); ${ }^{\mathrm{d} D e p a r t m e n t ~ o f ~}$

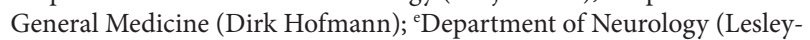
Ann Hall), Flinders Medical Centre, South Australia, Australia

Conflict of Interest: None

Correspondence to: Dr Alex Barnes, Department of Gastroenterology, Flinders Medical Centre Flinders Drive, Bedford Park, SA 5042,

Australia, e-mail: alex.barnes@sa.gov.au

Received 6 November 2020; accepted 30 November 2020; published online 27 January 2021

DOI: https://doi.org/10.20524/aog.2021.0582 3-week history of intermittent bloody diarrhea, odynophagia, general malaise, and subjective fever. She had no pertinent medical history other than her multiple sclerosis managed with ocrelizumab, initiated 18 months prior to admission with 3 infusions to date. She was a non-smoker. Family history included a brother with Crohn's disease. Examination findings included mild tenderness over the lower abdomen, and mild abdominal distension. Her heart rate was elevated at $109 \mathrm{bpm}$, the patient was normotensive 135/80 $\mathrm{mmHg}$, afebrile, and saturating well on room air.

Initial investigations revealed hemoglobin $107 \mathrm{~g} / \mathrm{L}$, C-reactive protein (CRP) $253.9 \mathrm{mg} / \mathrm{dL}$, albumin $30 \mathrm{~g} / \mathrm{L}$, and white cell count in the normal range, with lymphocytes $0.6 \times 10^{9} /$ L. Flow cytometry on serum could not detect any CD20+ or CD19+ cells. Stool testing for pathogenic bacteria including Clostridium difficile toxin, viruses, and parasites was negative. Herpes simplex virus (HSV) IgG/IgM viral serology was negative. Cytomegalovirus (CMV) IgG was positive, and IgM negative. Supplementary investigations including echocardiogram and computed tomography of chest, abdomen and pelvis showed no other contributing pathology.

An esophagogastroduodenoscopy (EGD) showed deep, punched-out ulcerations of the esophagus, and flexible sigmoidoscopy showed scattered deep ulcerations with severe inflammation to the point of insertion into the proximal transverse colon (Fig. 1). Colonic biopsy histology showed patchy, moderately-active chronic inflammation with cryptitis and crypt abscess formation with architectural disturbance, without granulomas. Immunohistochemistry could not detect any CD20+ cells. Esophageal biopsies demonstrated ulcerated squamous mucosa without viral inclusions or granulomas. Immunohistochemistry and tissue polymerase chain reaction testing of colonic and esophageal biopsies was negative for CMV and HSV. EGD and flexible sigmoidoscopy were repeated 3 days later which demonstrated similar endoscopic findings and histology with no evidence of CMV or HSV infection. 

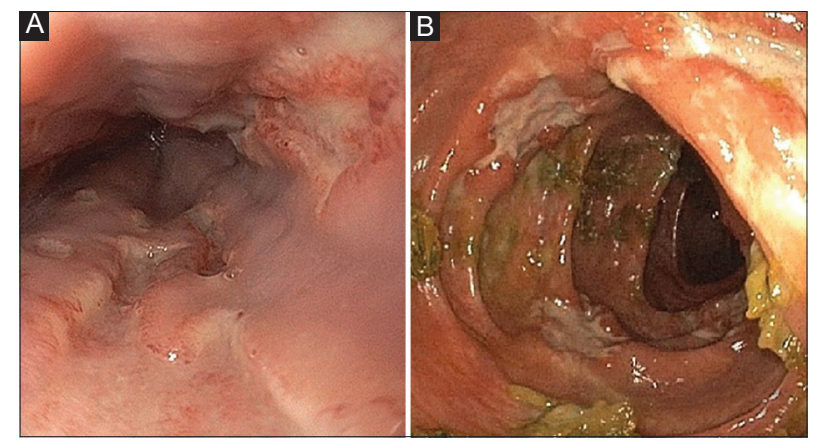

Figure 1 Endoscopic images of the esophagus (A) showing deep punched out ulcerations and from the left colon (B) showing scattered deep ulcerations

The patient was assessed as having likely ocrelizumabinduced colitis and esophagitis, that may represent new onset Crohn's disease. She was commenced on intravenous (iv) hydrocortisone $100 \mathrm{mg}$ q.i.d., along with oral pantoprazole and sucralfate for symptomatic relief. Odynophagia resolved within $48 \mathrm{~h}$, with disappearance of blood and resolution of diarrhea following $70 \mathrm{~h}$ of iv hydrocortisone. This was paralleled by a significant reduction in inflammatory markers with CRP improving to $12.6 \mathrm{mg} / \mathrm{L}$ following 1 week of treatment. She was discharged on a tapering dose of oral prednisolone. No further doses of ocrelizumab were planned.

Repeat colonoscopy 3 months following presentation showed resolution of active inflammatory changes in the esophagus and colon with post-inflammatory polyps seen in the colon. Histology from colonic biopsies showed mild active chronic colitis with development of an intraepithelial lymphocytosis commonly seen with microscopic colitis. Immunohistochemistry on colonic biopsies showed the presence of CD20+ cells. Serum lymphocyte subsets showed $\mathrm{CD} 19+$ count had recovered into the normal range $(0.20 \mathrm{x}$ $\left.10^{9} / \mathrm{L}\right)$. Repeat colonoscopy 9 months later was unremarkable with random colonic biopsies within normal limits.

\section{Discussion}

Regulatory B-cell depletion has been associated with colitis in mouse models and has also been demonstrated in peripheral blood and intestinal tissue of patients with ulcerative colitis $[4,5]$. The role of regulatory B-cells in maintaining gut homeostasis and suppressing the development of colitis may also explain the failure of anti-CD20 agents, such as rituximab, in treating inflammatory bowel disease (IBD).

Rituximab has previously been associated with de novo IBD and microscopic colitis [6]. Eckmann et al reported a 5\% incidence of rituximab-induced colitis with median time to onset of diarrhea of 20 months post first dose with most experiencing resolution in the months following drug withdrawal [6], similar to the time frame in this case. Sunjata et al reported a case of severe ocrelizumab colitis that relapsed after initially responding to iv corticosteroids resulting in colectomy [3]. Consideration could be given to an entity of anti-CD20-induced colitis that may lead to a self-limited IBD-like presentation through depletion of regulatory B-cells and may respond to IBD treatment.

This case prompted multi-disciplinary discussion regarding the use of additional biologic agents to treat IBD in the setting of multiple sclerosis, particularly given the potential compounding risk of serious and opportunistic infections. Tumor necrosis factor- $\alpha$ inhibitors have been reported to exacerbate demyelinating diseases [7], and their use in patients with multiple sclerosis is contraindicated. Consideration was given to the use of the gut-specific anti-integrin inhibitor vedolizumab, although patients on this agent are still being closely monitored for the development of progressive multifocal leukoencephalopathy given its similarity to natalizumab $[8,9]$. Ustekinumab, an interleukin (IL) -12/IL-23 antagonist, is not known to have any additional risk of malignancy and infection, nor high frequency neurological adverse effects [10], and was considered as a potential escalation therapy.

In conclusion, ocrelizumab-induced B-cell depletion has been shown to lead to improved outcomes in primary progressive multiple sclerosis. Similar to previously documented cases with rituximab, another anti-CD-20 antibody, our case demonstrated the development of an IBD-like illness following ocrelizumab-induced B-cell depletion. The development of a diarrheal illness on ocrelizumab should prompt consideration of an IBD-like illness and cautious endoscopic examination. Reporting of further cases of the development of an IBD-like phenotype in patients exposed to ocrelizumab is encouraged.

\section{References}

1. Hauser SL, Bar-Or A, Comi G, et al; OPERA I and OPERA II Clinical Investigators. Ocrelizumab versus interferon beta-1a in relapsing multiple sclerosis. N Engl J Med 2017;376:221-234.

2. Montalban X, Hauser SL, Kappos L, et al; ORATORIO Clinical Investigators. Ocrelizumab versus Placebo in Primary Progressive Multiple Sclerosis. N Engl J Med 2017;376:209-220.

3. Sunjaya DB, Taborda C, Obeng R, Dhere T. First case of refractory colitis caused by ocrelizumab. Inflamm Bowel Dis 2020;26:e49.

4. Wang L, Ray A, Jiang X, et al. T regulatory cells and B cells cooperate to form a regulatory loop that maintains gut homeostasis and suppresses dextran sulfate sodium-induced colitis. Mucosal Immunol 2015;8:1297-1312.

5. Xinrui Wang, Yonggang Zhu, Manli Zhang, et al. Ulcerative colitis is characterized by a decrease in regulatory B cells. J Crohns Colitis 2016;10:1212-1223.

6. Eckmann JD, Chedid V, Quinn KP, Bonthu N, Nehra V, Raffals LE. De novo colitis associated with rituximab in 21 patients at a tertiary center. Clin Gastroenterol Hepatol 2020;18:252-253.

7. Mohan N, Edwards ET, Cupps TR, et al. Demyelination occurring during anti-tumor necrosis factor alpha therapy for inflammatory arthritides. Arthritis Rheum 2001;44:2862.

8. Sandborn WJ, Feagan BG, Rutgeerts P, et al. GEMINI 2 Study Group. Vedolizumab as induction and maintenance therapy for Crohn's disease. N Engl J Med 2013;369:711.

9. Clerico M, Artusi CA, Liberto AD, et al. Natalizumab in multiple sclerosis: long-term management. Int J Mol Sci 2017;18:940.

10. Sandborn WJ, Gasink C, Gao LL, et al; CERTIFI Study Group. Ustekinumab induction and maintenance therapy in refractory Crohn's disease. N Engl J Med 2012;367:1519-1528. 